\title{
Across-stage consequences of thermal stress have trait-specific effects and limited fitness costs in the harlequin ladybird, Harmonia axyridis
}

\author{
Rebecca Shinner $^{1} \cdot$ John S. Terblanche ${ }^{2} \cdot$ Susana Clusella-Trullas $^{1}$ (D)
}

Received: 16 October 2019 / Accepted: 21 April 2020

(c) Springer Nature Switzerland AG 2020

\begin{abstract}
The fitness consequences of thermal plasticity of functional traits of insects, and the life stages at which these responses manifest, remain a subject of much debate. Here, we examine whether a short-term exposure to cold, medium or warm fluctuating temperature regimes during development (larval or adult) affects adult performance, thermal tolerance and fitness of the globally invasive harlequin ladybird Harmonia axyridis. We found plastic responses of a metric of heat stress resistance-critical thermal maximum-and of preferred body temperature after adult temperature exposure, but not in other traits measured. By contrast, exposure of larval stages resulted in plasticity of adult walking performance (height and breadth of the curve) but not tolerance or preference. We found distinct fitness responses between larval and adult treatments, but a composite fitness index revealed negligible effects on reproductive output. These results suggest that the drivers underlying the plasticity of temperature tolerance and temperature selection are different to those shaping the plasticity of walking speed. By testing specific predictions based on current theory of developmental and reversible plasticity, this study contributes novel data to plastic responses of behaviour, stress resistance and fitness to temperature exposure across life stages and thus, provides insights to the broader evolutionary and ecological significance of these responses.
\end{abstract}

Keywords Beneficial acclimation $\cdot$ Fitness costs $\cdot$ Ontogeny $\cdot$ Invasive species $\cdot$ Heat waves

Electronic supplementary material The online version of this article (https://doi.org/10.1007/s 1068 2-020-10045-1) contains supplementary material, which is available to authorized users.

Susana Clusella-Trullas

sct333@sun.ac.za

1 Centre for Invasion Biology, Department of Botany and Zoology, Stellenbosch University, Stellenbosch 7602, South Africa

2 Centre for Invasion Biology, Department of Conservation Ecology and Entomology, Stellenbosch University, Stellenbosch 7602, South Africa 


\section{Introduction}

Phenotypic plasticity can facilitate the persistence of organisms in changing environments and thus, be adaptive when the responses maintain performance and enhance fitness in the novel conditions (Levins 1968; West-Eberhard 2003; Ghalambor et al. 2007). Thermal acclimation, as a form of phenotypic plasticity, is expressed as a compensatory response after a short-term temperature exposure and is beneficial if it maintains or improves the fitness of an organism despite potential costs (Via and Lande 1985; West-Eberhard 2003; Bowler 2005; Sørensen et al. 2016). For example, changes in the magnitude of daily temperature fluctuations can result in increased stress resistance, likely preparing individuals to upcoming seasonal shifts or extremes (Sgrò et al. 2016). This plasticity is generally referred to as reversible acclimation and takes place during the mature stage of the organism. By contrast, developmental acclimation is widely viewed as irreversible and results from temperature experienced during development having persistent effects on subsequent stages (e.g. from larvae to mature adults in insects) (Piersma and Drent 2003; Wilson and Franklin 2002). Multiple studies have assessed the extent of temperature acclimation responses of insects at the adult stage but fewer examine the long lasting effects of larval exposures to performance and fitness of adults, especially in non-Drosophila models (Drosophila: e.g. Gibert et al. 2001; Cavieres et al. 2016; Kellermann et al. 2017; but see e.g. tsetse flies: Terblanche and Chown 2006; butterflies: Zeilstra and Fischer 2005; Fischer et al. 2003).

Theoretically, reversible acclimation should be favoured in environments that vary within a generation so that the fast phenotypic response enables maintenance of fitness in the face of this variation (e.g. Gilchrist 1995). Developmental plasticity can enhance responsiveness later in life but can also impose costs (e.g. opportunity cost; Weinig and Delph 2001) or compromise performance if the conditions experienced early in life differ from those later in life (phenotype-environment mismatch) (Cotto et al. 2019). Developmental plasticity can be favoured in environments that vary within-generations but do so predictably, so that the conditions encountered by early stages signal the environmental mean that other stages or adults may face later in life (Beaman et al. 2016). Alternatively, non-adaptive or maladaptive plasticity can manifest as phenotypic responses that are opposite to those that would be favoured by selection, and may stem from genetic correlations or relaxed selection (Ghalambor et al. 2007; Cotto et al. 2019). However, what may appear non-adaptive at one stage may be adaptive later in the life cycle, or may have evolved under multiple environmental cues (Chevin and Lande 2015). Therefore, these responses may have not been tested or modelled under the appropriate conditions or at the relevant time in the life cycle (Potter et al. 2011; Nougué et al. 2016; Sørensen et al. 2016; Uno and Stillman 2020).

Insects possess complex life cycles with multiple different stages in which size, behaviour and mobility can vary greatly (e.g. immobile egg and less mobile pupal stages versus mobile larval and adult stages) and influence the extent of physiological and behavioural plasticity (Fusco and Minelli 2010; Kingsolver et al. 2011; Lockwood et al. 2018). Across life stages, exposure to variation in temperature may not necessarily have an additive or straightforward predictable effect and acclimation responses may vary in their direction, magnitude and persistence (Rako and Hoffmann 2006; Terblanche and Chown 2006; Zhang et al. 2015; Slotsbo et al. 2016). These time-dependent responses all feed back into the magnitude and direction of natural selection on basal and plastic stress responses and may also depend upon any potential constraints and costs of plasticity (Pigliucci et al. 
2006; Schulte et al. 2011; Sgrò et al. 2016). Therefore, examining the fitness consequences of varying the timing of exposure across life stages may contribute to elucidating drivers of plasticity and potential costs and benefits of thermal acclimation (Bowler and Terblanche 2008; Sgrò et al. 2016).

The magnitude of plasticity can vary among traits despite potential genetic correlations (both among different traits and between basal traits and their plasticity) (Kellermann et al. 2009, 2013; Fragata et al. 2015; Liefting et al. 2015; Hangartner and Hoffmann 2016). For example, the plasticity of upper thermal tolerance is typically lower than that of lower thermal tolerance, both within and between species (Gaston and Chown 1999; Clusella-Trullas and Chown 2014; Sørensen et al. 2016). Therefore, assessing multiple traits, including distinct parameters of thermal performance curves, can highlight asymmetric effects of acclimation to high and low temperatures and potential trade-offs (Seebacher et al. 2005; Rako and Hoffmann 2006; Clusella-Trullas et al. 2010; Gerken et al. 2016; Slotsbo et al. 2016). How these effects change or are maintained when comparing the consequences of acclimation at different stages into adulthood has been poorly explored empirically (despite theory increasingly adding required levels of complexity, e.g. Westneat et al. 2019).

In this study, we aim to evaluate whether a short-term exposure to three temperatures during development impacts several measures of adult performance and how these responses compare to similar exposures experienced at the adult stage. To achieve this, we examined several performance traits (walking speed, temperature selected in a thermal gradient and temperature tolerance limits) as well as diverse fitness proxies (relative egg production, hatching success and developmental time) of adult harlequin ladybirds, Harmonia axyridis (Pallas) (Coleoptera: Coccinellidae), exposed to low, medium or high temperature fluctuating environments at the larval or early adult stages. These regimes were based on temperatures encountered in natural conditions but also well-established effects of temperature on development, reproduction and growth in this species, which often peaks between 20 and $25^{\circ} \mathrm{C}$ (Lamana and Miller 1998; Roy et al. 2016; Zhang et al. 2014). The physiological and behavioural traits measured here are thought to be ecologically and evolutionary meaningful for diverse reasons, are widely adopted in climate adaptation studies, and well correlated with various geographical environmental patterns (e.g. Andersen et al. 2015; Chown and Terblanche 2007). Native to Asia, Harmonia axyridis has successfully invaded many regions of the world, encompassing diverse climates (Roy et al. 2016) and phenotypic plasticity, including of colour variation, has been proposed to contribute to its persistence in novel areas (Grill et al. 1997; Michie et al. 2010; Purse et al. 2015). In South Africa, H. axyridis was first detected in 2001 in the southern Western Cape region which has a cool, mesic climate (Roy et al. 2016). In this area, H. axyridis is multivoltine and has four distinct stages: egg, larvae (4 instars), pupa and adult. It is active most of the year, except during the very dry summer months and parts of winter when it forms large aggregations. While larvae typically reside within single or a few neighbouring plants, adults are more mobile and disperse readily by flight, covering longer distances to find oviposition sites (Kawai 1976; Osawa 2000). Longevity for non-hibernating adults ranges from 22 to 150 days (Berkvens et al. 2008; Raak-van den Berg et al. 2017) and several studies refer to its long-distance dispersal capacity (Brown et al. 2011; Lombaert et al. 2014).

Here we follow theoretical expectations that propose plasticity is favoured when the environmental factors affecting fitness vary on appropriate time scales: (1) if the environment is predictable across life stages, the phenotypic change elicited by an exposure in early stages should be beneficial to later stages, (2) if the environment is not predictable and highly variable, development may buffer this unpredictability with no effects on the adult stage and adult reversible plasticity may be favoured. Given the relatively long life span of 
this species and different movement patterns across life stages, we may expect greater support for (2). However, we recognize that substantial complexity is likely to underlie these responses (Beaman et al 2016; Sgrò et al. 2016; Westneat et al. 2019), potentially limiting the robustness of these predictions. We also measure downstream fitness consequences of developmental and adult temperature exposures using a suite of life-history traits and test whether plasticity of locomotion performance, thermal preference and tolerance, if any, is associated to adult fitness components.

\section{Materials and methods}

\section{Field collections and maintenance}

Adult Harmonia axyridis (form succinea, $\mathrm{n}=250$ ) were collected from several rose gardens in Stellenbosch, Western Cape Province, South Africa $\left(33.9200^{\circ} \mathrm{S}, 18.8600^{\circ} \mathrm{E}\right)$ from March to July 2013 and 2014, and October to November 2013. The stock population was maintained in 2 -L plastic containers at similar low densities ( $\mathrm{n} \leq 40$ per container), lined with paper towels and given shredded paper for refugia. Containers were kept in temperature-controlled incubators (MIR-153, Sanyo, Japan) at cycles of $18 \mathrm{~h}$ at $25.3 \pm 0.4{ }^{\circ} \mathrm{C}$ and $6 \mathrm{~h}$ at $18.3 \pm 1.2{ }^{\circ} \mathrm{C}$ (mean temperature $23.5 \pm 3.1{ }^{\circ} \mathrm{C}$; photoperiod $14 \mathrm{~L}: 10 \mathrm{D}$ ). Distilled water and a solution of 1:10 honey:distilled water were available at all times on cotton wool. Aphids (common rose aphids (Macrosiphon rosae, Homoptera: Aphididae), oak aphids (likely Myzocallis castanicola) and lab-reared Russian wheat aphids (Diuraphis noxia, Homoptera: Aphididae) were provided ad libitum every third day. First generation $\left(\mathrm{G}_{1}\right)$ eggs were reared in Petri dishes lined with filter paper until reproductive maturity ( 10 days, Lanzoni et al. 2004) and then placed into 2-L containers with the same conditions as the $G_{0}$ adults. $G_{2}$ eggs were transferred into Petri dishes and $1^{\text {st }}$ instar larvae divided into groups of $n=10 \pm 2$ individuals one day after hatching. All larvae $\left(\mathrm{G}_{1}\right.$ and $\left.\mathrm{G}_{2}\right)$ were fed daily (1:10 honey:water solution and common rose aphids). $G_{2}$ individuals were placed individually in Petri dishes on the day they emerged from pupae and kept in the same conditions as larvae except that aphids were only offered every third day.

\section{Temperature treatments}

Three temperature cycles: 'Cold' $\left(20.2 \pm 0.2{ }^{\circ} \mathrm{C} 18 \mathrm{~h}\right.$ day and $13.7 \pm 1.1{ }^{\circ} \mathrm{C} 6 \mathrm{~h} \mathrm{night}$, mean $\left.=18.6 \pm 2.8^{\circ} \mathrm{C}\right)$, 'Medium' $\left(25.3 \pm 0.4{ }^{\circ} \mathrm{C} 18 \mathrm{~h}\right.$ day and $18.3 \pm 1.2{ }^{\circ} \mathrm{C} 6 \mathrm{~h}$ night, mean $=23.5 \pm 3.1^{\circ} \mathrm{C}$ ) and 'Warm' $\left(30.4 \pm 0.4{ }^{\circ} \mathrm{C} 18 \mathrm{~h}\right.$ day and $23.5 \pm 0.5{ }^{\circ} \mathrm{C} 6 \mathrm{~h}$ night, mean $=28.6 \pm 3.0^{\circ} \mathrm{C}$ ) were controlled by three incubators (Sanyo, MIR-153, Japan), verified using hygrochron iButtons (Model DS1923, Maxim, USA) and set with a photoperiod of 14L:10D for all treatments. Petri dishes containing $\mathrm{G}_{2}$ larvae were randomly assigned to a temperature treatment either during development (from third instar to pupation) or adulthood (which started 11 days after emergence from pupae to ensure reproductive maturity). The third and fourth larval stages were chosen for the exposure as these are typically most sensitive to carry-over effects in this species (Knapp and Nedved 2013; Knapp 2014) and other species (e.g. Kooi and Brakefield 1999). This is likely due to adults experiencing conditions more similar to late immature stages compared to earlier ones. Body mass was recorded for $\mathrm{n}=3$ larvae per Petri dish at the beginning of the larval exposures and again on the first day of reaching the fourth instar, and adults were weighed on the first and last 
days of temperature treatments. Handling was kept constant for larval and adult treatment groups.

The duration of adult temperature treatments was between 7 and 15 days to allow enough time for thermal acclimation (Weldon et al. 2011; Knapp and Nedvěd 2013), while also ensuring that individuals were tested within a relatively constant age-window (18-25 days from pupae emergence). Adult beetles were only used once for all experimental manipulations, and were tested within the same age range regardless of whether they were exposed to temperature treatments as larvae or adults.

For fitness trials, 18-25 day old male and female beetles originating from larval or adult acclimation groups were paired in individual petri dishes and placed in the 'Medium' treatment. Paired beetles ( $n=10$ per group) were fed common rose aphids every third day and honey solution every day while their offspring were fed aphids and honey solution every day (see Field collections and maintenance).

\section{Thermal preference}

The thermal gradient consisted of nine Perspex rods dividing a metal platform into ten lanes $(75 \times 2 \mathrm{~cm})$, that had a heating element (Shinko Technos Co. Ltd Overseas Division, JCS-33A, Japan) set to $40{ }^{\circ} \mathrm{C}$ at one end and circulating water at a temperature controlled by a $50 \%$ water, $50 \%$ glycol bath (Grant, GP200, England) set to $0{ }^{\circ} \mathrm{C}$ at the other end. A fixed Perspex lid, marked with horizontal lines every $2 \mathrm{~cm}$, had a removable transparent section in the centre of the gradient to enable the introduction and removal of animals. Masking tape was positioned down the centre of each lane to ensure the beetles had adequate traction for walking. Fluon (Fluon ${ }^{\circledR}$ AD208E, England) was painted on the walls of each lane to prevent beetles from straying up the walls and thus escaping or modifying the experienced thermal gradient. Trials were conducted between 09:00 and 17:00 in a laboratory with both artificial and natural light to ensure an even light intensity across the thermal gradient.

Temperatures in the gradient were recorded for each trial using iButtons (Maxim Hygrochron iButtons, DS1922L, USA) distributed along the length and width of the gradient. Preliminary trials showed minimal variation in temperature among lane positions (ranging from a SD of $0.2{ }^{\circ} \mathrm{C}$ at the cold end to $0.9^{\circ} \mathrm{C}$ at the warm end), and indicated that the setup produced a highly stable $11-40{ }^{\circ} \mathrm{C}$ linear gradient. In addition, operative temperatures $\left(\mathrm{T}_{\mathrm{e}}\right)$, defined as the steady-state temperatures of an organism in a particular microclimate in the absence of metabolic heating and evaporative cooling (Bakken 1992), were mapped on the gradient by monitoring the temperature of freshly killed adult individuals placed at regular intervals along the gradient. Fine copper-constantan (Type T, 36 gauge) thermocouples were inserted into the thoraxes of 16 beetles placed along the gradients and connected to a datalogger (PICO Technology, TC-08 Thermocouple Datalogger, UK). Operative temperatures were temporally stable and showed a strong linear relationship with the position on the gradient similar to that recorded by the iButtons (see Supplementary Material, Fig. S1). We therefore used the iButton linear relationship with position recorded for each trial to infer body temperature given the individuals' positions along the gradient.

For each trial, five adults were placed individually in alternate lanes at the centre of the thermal gradient and allowed a 15 min settling period. Their position was then determined every five minutes for two hours using video footage recorded with a webcam (Logitek, HD C270, Switzerland). All individuals had distilled water available but were fasted for 20-26 h prior to trials to avoid potential post-feeding effects (e.g. McCue et al. 2016). 
Body mass was recorded to the nearest $0.001 \mathrm{~g}$ on an electronic microbalance (Avery Berkel, UK) before and after each trial. In total, 219 individuals were tested, but those that did not move $(\mathrm{N}=20,<10 \%)$ in the gradient were removed from the dataset as no position was actively selected. This resulted in a minimum of 30 (15 males, 15 females) from each adult and larval temperature treatment. In addition, to rule out a cold-induced lack of thermoregulation (Dillon et al. 2012) or account for other biases (e.g. the gradient being an unnatural environment) or innate dispersal tendencies on the gradient, three control trials were carried out with the stage held at a constant temperature of $15{ }^{\circ} \mathrm{C}, 25^{\circ} \mathrm{C}$ or $35^{\circ} \mathrm{C}$ in a temperature-controlled climate room. The protocol was the same as that described for the gradient trials, but a smaller sample size of at least 10 (5 males, 5 females) from each of the adult and larval temperature treatments were used for each control trial (total $N=194$ ).

\section{Critical thermal limits}

Adults were placed individually in Perspex chambers positioned in series in a double-jacketed system connected to a programmable water bath (Grant, GP200, UK) which regulated the temperature of water flowing through the system. A copper-constantan thermocouple connected to a datalogger (PICO Technology, TC-08 Thermocouple Datalogger, UK) was placed in the central chamber to monitor the temperature throughout trials. After $15 \mathrm{~min}$ of equilibration to the starting temperature $\left(25^{\circ} \mathrm{C}\right)$, the temperature was either increased (for critical thermal maximum, $\mathrm{CT}_{\max }$ ) or decreased (for critical thermal minimum, $\mathrm{CT}_{\text {min }}$ ) at a rate of $0.05{ }^{\circ} \mathrm{C} \cdot \mathrm{min}^{-1}$ as this reasonably approximates the natural diurnal heating and cooling rates measured at ground and tree-level microsites in Stellenbosch (e.g. Nyamukondiwa and Terblanche 2010). Pilot trials showed that beetles' body temperature rapidly equilibrated to the environmental temperature (Fig. S2), indicating that the temperature recorded by the thermocouple could be used as a reliable proxy for body temperature. Individuals were monitored every hour initially, then every $2-3$ min as signs of heat stress became apparent for $\mathrm{CT}_{\max }($ after $\sim 5 \mathrm{~h}$ ), and every 6-7 min as chill coma onset neared for $\mathrm{CT}_{\min }\left(\right.$ after $\sim 6.5 \mathrm{~h}$ ). $\mathrm{CT}_{\max }$ was recorded as the temperature at which individuals lost coordination and were unable to grab and/or cling to a fine paintbrush; $\mathrm{CT}_{\min }$ was recorded as the temperature at which there was loss of coordinated movement and movement of the head, legs and antennae ceased even after gentle stimulation with a fine paintbrush. All tests were carried out by a single observer (R.S.) to eliminate observer bias and started between 07:30 and 10:30 to prevent potential diurnal effects. All individuals were fasted for 18-22 h before trials and body mass was recorded before and after each trial. In total, at least 30 individuals from each temperature treatments and life stage combination (15 males, 15 females) were tested for both $\mathrm{CT}_{\max }(\mathrm{n}=204)$ and $\mathrm{CT}_{\min }(\mathrm{n}=197)$.

\section{Walking speed}

Adults were tested in a Perspex custom-made unit connected to a water bath (Grant, GP200, England) which maintained a flow of water along the base and sides of ten lanes ( $1 \mathrm{~cm} \times 1 \mathrm{~cm} \times 22 \mathrm{~cm}$ per lane). Each lane was lined with paper along the base to provide a uniform surface with good traction. The top of the unit was marked every centimetre with lines perpendicular to the direction of the lanes to provide a reference for distance moved. Each individual was tested at seven temperatures $\left(0,10,20,30,40,45\right.$ and $\left.50^{\circ} \mathrm{C}\right)$ over a two day period; the first and last temperatures tested were $0{ }^{\circ} \mathrm{C}$ and $50{ }^{\circ} \mathrm{C}$ respectively, but the intermediate temperatures were randomised to avoid any potential systematic 
effects of heating or chilling-related injury. The operative temperature $\left(\mathrm{T}_{\mathrm{e}}\right)$ for each trial was recorded every second using a data logger (TC-08, Pico Technology, UK) and thin thermocouples inserted in freshly killed beetles positioned $3 \mathrm{~cm}$ from each end of lanes 2 , 6 and 10 and averaged over a five minute period during which each trial took place.

Individual adults were placed in alternate lanes and allowed to equilibrate to the temperature for $5 \mathrm{~min}$ before the trial began. They were initially stimulated to walk by gently moving a small paintbrush on the paper base just behind the beetle. Individuals completed four runs at each test temperature, of which the fastest three were used to calculate a mean walking speed. Runs were judged as usable if the individuals walked $>3 \mathrm{~cm}$ (i.e. $>5$ times its body length of $6 \mathrm{~mm}$, Roy et al. 2016) continuously on the surface plane. Individuals were weighed before and after each test temperature to monitor body condition and allowed to recover in their host incubator with distilled water for at least one hour between test temperatures. Trials were filmed from above using a digital webcam (Logitek, HD C270, Switzerland). Videos were analysed with MaxTraq Standard motion tracking software (Innovision Systems, USA). The run was judged to start at the frame in which the head of the individual crossed the first line after being poked, and ended at the frame in which the head crossed the final line before stopping. The distance travelled was divided by time to the nearest $0.015 \mathrm{~s}$ to give speed (in $\mathrm{cm} / \mathrm{s}$ ). A total of 120 individuals were tested, 20 (10 males, 10 females) from each adult and larval temperature treatment.

\section{Fitness estimates}

The number of egg clutches and the total number of eggs deposited by 10-15 pairs per treatment was monitored daily for 70 consecutive days since initial female-male pairing. The first three egg batches were placed in separate petri dishes and eggs monitored daily for the number of hatched larvae. The following variables were recorded: female body mass, number of eggs per clutch, hatching success and offspring developmental time from egg to adult.

\section{Statistical analysis}

Prior to analyses, data were checked for outliers and three data points were removed (one out of $205 \mathrm{CT}_{\max }$ and two out of 120 walking speeds). To test if there were consistent mass effects between life stage exposures and disentangle these from sex effects, we use general linear models with body mass (measured immediately prior to testing) as a response variable, and Stage, Sex and Treatment as categorical predictors.

$\mathrm{CT}_{\max }$ data were first analysed for each stage group (adult or larval exposures) separately to test for the effects of acclimation on the $\mathrm{CT}_{\max }$ of adults. We used a general linear model with $\mathrm{CT}_{\max }$ as the response variable, and the categorical variables Treatment (temperature treatments) and Sex and the continuous variable Mass (at the start of the trial) as predictors. $\mathrm{CT}_{\min }$ data (transformed to $\mathrm{n}+1$, to avoid numbers $<0$ ) were analysed using a generalized linear model assuming a Gaussian distribution and $\log$ link function, with the same predictors as described for the $\mathrm{CT}_{\max }$ model above. Model overdispersion was checked but no corrections were necessary.

For $\mathrm{T}_{\text {pref }}$ data, we first ran a Kolmogorov-Smirnov test to assess whether the distribution of individuals in the thermal gradient differed from that of the control trials. A general linear model was then used to assess the effects of Treatment, Sex and Mass on the response variable $\mathrm{T}_{\text {pref }}$ (mean over the $2 \mathrm{~h}$ trial) for each stage group separately. The 
outcomes of these models were similar when the mean, median or mode of body temperatures was used as $\mathrm{T}_{\text {pref }}$.

For walking speed, $\mathrm{T}_{\mathrm{opt}}$ and $\mathrm{U}_{\max }$ were extracted using TableCurve2D curve-fitting software (https://www.sigmaplot.com/products/tablecurve2d/Tablecurve2d.php) (as in e.g. Lachenicht et al. 2010). Asymmetric curve equations were fitted (maximum of 100 iterations) and sorted by degrees of freedom-adjusted $\mathrm{R}^{2}$ values to select the best-fit curve with the least model parameters. In five cases, this method yielded biologically unrealistic curves, so either the second-ranked curve was selected or, if still inappropriate, the process was re-run but with asymmetric equation option deselected. In addition, the performance breadth $\left(\mathrm{T}_{\mathrm{BR}}\right.$ ) was calculated using Gilchrist's (1996) method:

$$
T_{b r}=\sqrt{\sum\left(\frac{u_{i}\left(T_{i}-T_{o p t}\right)}{u_{\max }}\right)^{2}}
$$

where $T_{b r}$ is an index, analogous to the second moment of area about a neutral axis, where $u_{i}$ is walking velocity at temperature $T_{i}$. This index describes the distribution of relative velocity about a central point, in this case around $\mathrm{T}_{\text {opt }}$; the velocities, $u_{i}$, are standardized to $U_{\max }$ to remove spurious correlations between $T_{B R}$ and $U_{\max }$ (Gilchrist 1996). General linear models were then used to analyse each parameter, using $\mathrm{T}_{\mathrm{opt}}, \mathrm{U}_{\max }$, and $\mathrm{T}_{\mathrm{BR}}$ of the performance curve as response variables and explanatory predictors as described for analysis of the $\mathrm{T}_{\text {pref }}$ data.

For fitness-related traits, we assessed the effect of stage and acclimation treatment on the number of egg clutches, the total number of eggs, the hatching success (number of hatched larvae relative to the number of eggs monitored from the first three clutches) and developmental time (from egg to adult emergence from pupa). The number of clutches and total number of eggs were analysed using a generalized linear model with poisson error distributions, and Mass (taken as body mass recorded at the start of the fitness assessment), Stage and Treatment as predictors (Treatment and Stage $=$ categorical, Mass $=$ continuous covariate). A quasi-poisson error distribution was used if overdispersion was found in these models. For hatching success, a generalized linear mixed model with binomial error distribution was used, with Treatment and Clutch number (i.e. first to third clutch) included as fixed factors and Individual and Clutch number included as random variables. Finally, we calculated a relative index of 'composite fitness' per female by multiplying the total number of eggs, the hatching success and the larval developmental success and divided by the time from egg-to-adult maturity. This allows estimation of a composite fitness index similar to that used in other studies (e.g. Overgaard et al. 2014). Essentially this fitness metric accounts for the temperaturedependence of development to give an estimate of instantaneous population growth rate (i.e. adjusting values of e.g. egg survival and number for how long it would take for them to hatch and reach maturity under a given condition). We assume that the production rate of, and time taken to replace, reproductive adults is a reasonable approximation of evolutionary fitness. This index was compared across treatments for each life stage using general linear models.

Finally, for all traits above, the effect of life stage acclimation on adult performance was assessed by repeating the models as above but including life stage exposure as a categorical predictor and not incorporating the Medium treatment which remained the same for both life stage exposures. Here we are interested in the overall mean change in the trait between life stage treatments only. 
All analyses were carried out in $\mathrm{R}$ (version 3.4.0). Step-wise model simplification to minimum adequate models was made by removing terms from the full model in order of least significance, with highest order interactions removed first. Models were compared using Akaike's Information Criteria before and after removing each term (following Crawley 2007). All models were assessed for robustness using residual vs. fitted values, normal QQ, scale-location and residuals vs. leverage plots as diagnostics. Statistics are reported from the summary function of linear models in $\mathrm{R}$ with appropriate contrasts used to compare across acclimation levels.

\section{Results}

For all traits investigated, male body mass was lower than female mass (Fig. S3). In addition, adults that were cold-acclimated as larvae were generally heavier than warm-acclimated ones, but this effect was not found in the adult treatments (Fig. S3). Therefore, both mass and sex were included in all statistical models.

\section{Critical thermal limits}

Larval exposures to temperature treatments did not significantly alter $\mathrm{CT}_{\max }$ of adults (Fig. 1a). By contrast, exposure to the Warm treatment in adulthood resulted in significantly higher $\mathrm{CT}_{\max }$ than either the Medium or Cold treatments $(t=4.14, p<0.01$; $t=3.95, p<0.01$ respectively), but $\mathrm{CT}_{\max }$ from Medium and Cold treatments did not differ $\left(t=-0.14, p=0.89\right.$, Fig. 1a). Mass was positively related to adult $\mathrm{CT}_{\max }$ in both larval and adult exposures $(\mathrm{t}=2.41, \mathrm{p}=0.02 ; t=2.48, p=0.015$, respectively).

Neither adult nor larval temperature treatments affected $\mathrm{CT}_{\min }$ (Fig. 1b). Mass was negatively related to adult $\mathrm{CT}_{\min }$ in both larval and adult exposures $(t=-2.04, p<0.05 ; t=-3.13$, $p<0.01$, respectively). Males $\left(1.05 \pm 0.09^{\circ} \mathrm{C}[\right.$ Mean $\left.\pm \mathrm{SE}]\right)$ had lower $\mathrm{CT}_{\min }$ than females $\left(1.37 \pm 0.09{ }^{\circ} \mathrm{C}[\mathrm{Mean} \pm \mathrm{SE}] ; t=-3.86, p<0.01\right)$ despite their smaller body size $(t=-6.36$, $p<0.01)$. Overall, $\mathrm{CT}_{\max }$ and $\mathrm{CT}_{\min }$ of adults acclimated at either adult or larval stages did not differ $\left(\mathrm{CT}_{\max }: t=-1.82, \mathrm{p}=0.07\right.$; CTmin: $\left.t=1.32, \mathrm{p}=0.19\right)$.

\section{Thermal preference}

In all treatment groups, the frequency distribution of beetle position in the thermal gradient differed significantly from controls which had the same set-up but without the thermal gradient (Fig. S4). Exposure to the Cold treatment during adulthood resulted in significantly higher $\mathrm{T}_{\text {pref }}$ than exposure to either the Medium or Warm treatments $(t=-4.19, p<0.01$; $t=-2.42, p=0.02$, respectively), but $\mathrm{T}_{\text {pref }}$ from Medium and Warm treatments did not differ $(t=1.65, p=0.11)$. By contrast, temperature treatments did not have a significant effect on $\mathrm{T}_{\text {pref }}$ when adults were exposed to treatments as larvae (Fig. 1c). Overall, $\mathrm{T}_{\text {pref }}$ of adults acclimated at the adult stage or larval stage did not differ $(t=-1.55, \mathrm{p}=0.12)$.

\section{Thermal performance curve and parameters}

Walking speed increased with test temperature between 0 and $50{ }^{\circ} \mathrm{C}$ in all groups, reaching $\mathrm{T}_{\text {opt }}$ between 42 and $45^{\circ} \mathrm{C}\left(\right.$ mean $\left.=43.5 \pm 2.5^{\circ} \mathrm{C}\right)$ before a sharp decline to zero by 

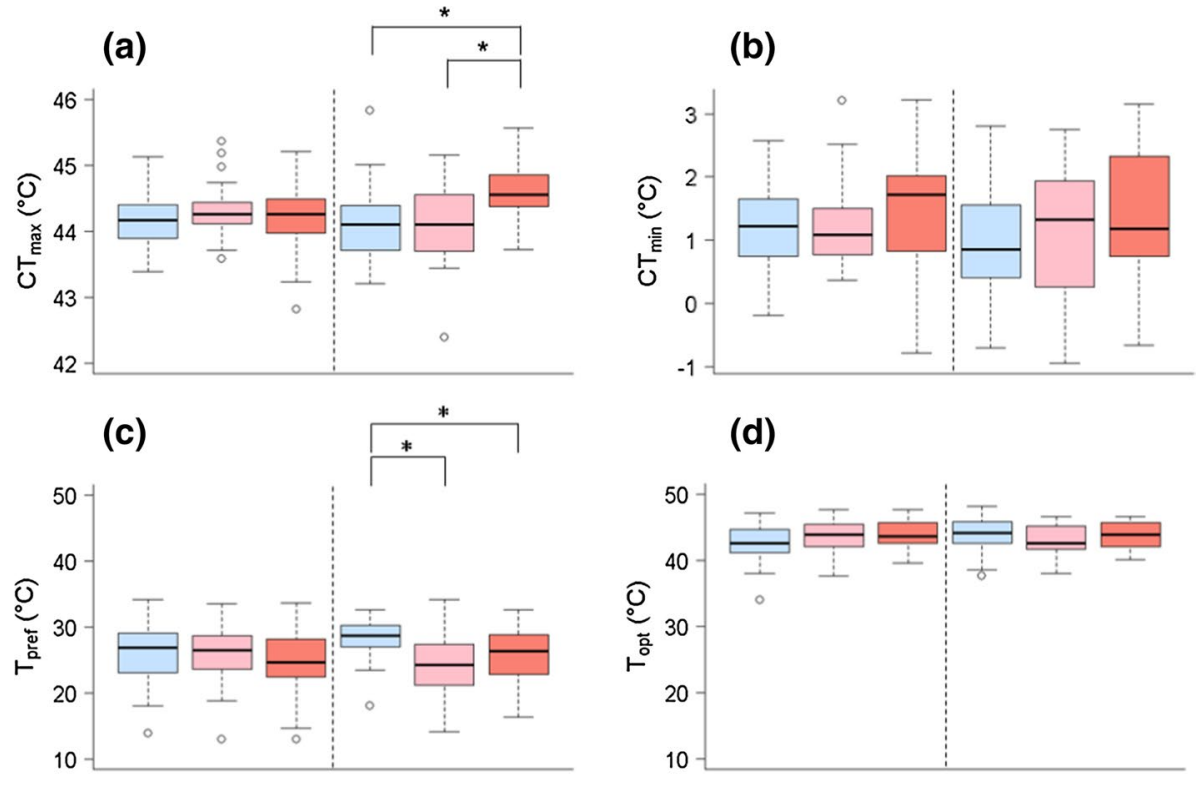

(d)

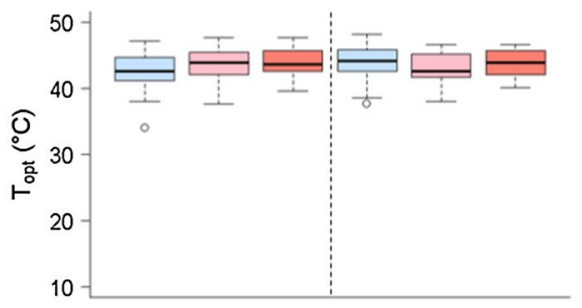

(e)
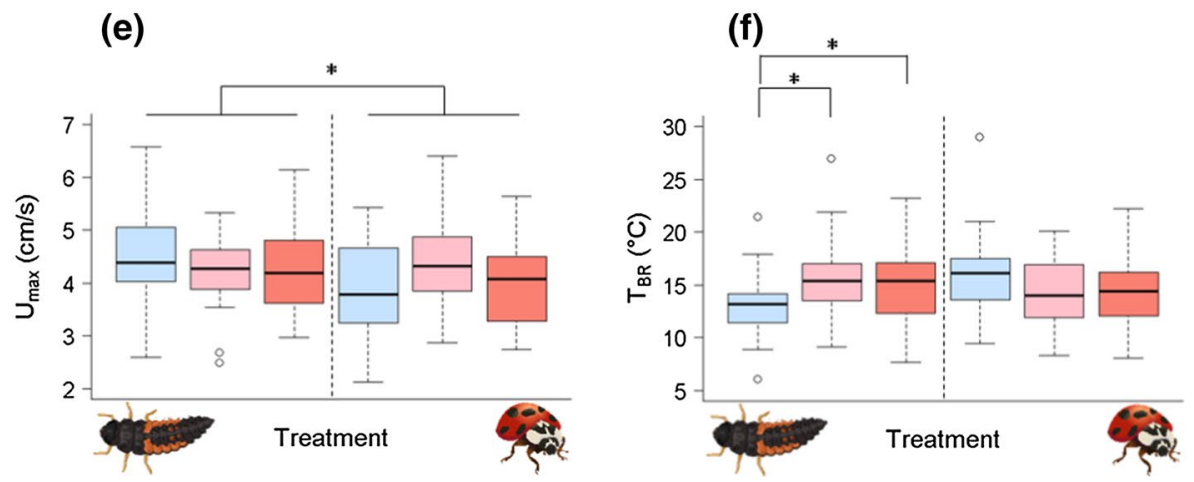

Fig. 1 Effects of larval and adult exposures to Cold, Medium and Warm temperature treatments (from left to right) on a $\mathrm{CT}_{\max } ; \mathbf{b} \mathrm{CT}_{\text {min }} ; \mathbf{c} \mathrm{T}_{\text {pref }} ; \mathbf{d} \mathrm{T}_{\mathrm{opt}} ; \mathbf{e} \mathrm{U}_{\mathrm{max}}$; and $\mathrm{f}_{\mathrm{BR}}$. Note: larval exposures to temperature treatments are shown on the left of each plot, adult exposures on the right; all individuals were tested for performance metrics during adulthood; significant differences are denoted with an asterisk. In all boxplots, the horizontal line represents the median, with the bottom and top of the box as the 25th and 75th percentiles respectively; the whiskers span 1.5 times the interquartile range and any outliers greater than or less than 1.5 times the interquartile range are plotted individually

$50{ }^{\circ} \mathrm{C}$ (Fig. 2). $\mathrm{T}_{\mathrm{opt}}$ did not overlap with $\mathrm{T}_{\text {pref }}$ (mean $=25.8 \pm 4.6^{\circ} \mathrm{C}$ ) for any of the treatment groups (Fig. 2).

Neither larval nor adult exposures to different temperature treatments had a significant effect on adult $\mathrm{T}_{\mathrm{opt}}$ (Fig. 1d) and there were no differences in adult $\mathrm{T}_{\mathrm{opt}}$ between life stage treatment groups $(\mathrm{t}=-0.84, p=0.41)$. Adult exposure to the Cold treatment resulted in a negative relationship between adult mass and $U_{\max }$ while the converse was found after exposure to Medium and Warm treatments $(\mathrm{t}=2.12, p=0.04 ; \mathrm{t}=2.19, p=0.03$ respectively; Fig. S5). Neither treatment nor interactions with mass affect adult $U_{\max }$ when beetles 
Fig. 2 Adult walking speed (mean \pm SE) at each test temperature after a) larval and b) adult exposure to Cold, Medium and Warm temperature treatments. Vertical bars are preferred body temperatures (width $=\mathrm{T}_{\text {pref }} \pm \mathrm{SE}$ ) for each treatment group. Black dashed line and white vertical bar $=$ Warm acclimation, pale grey line and bar=Medium acclimation, dark grey line and bar $=$ Cold acclimation
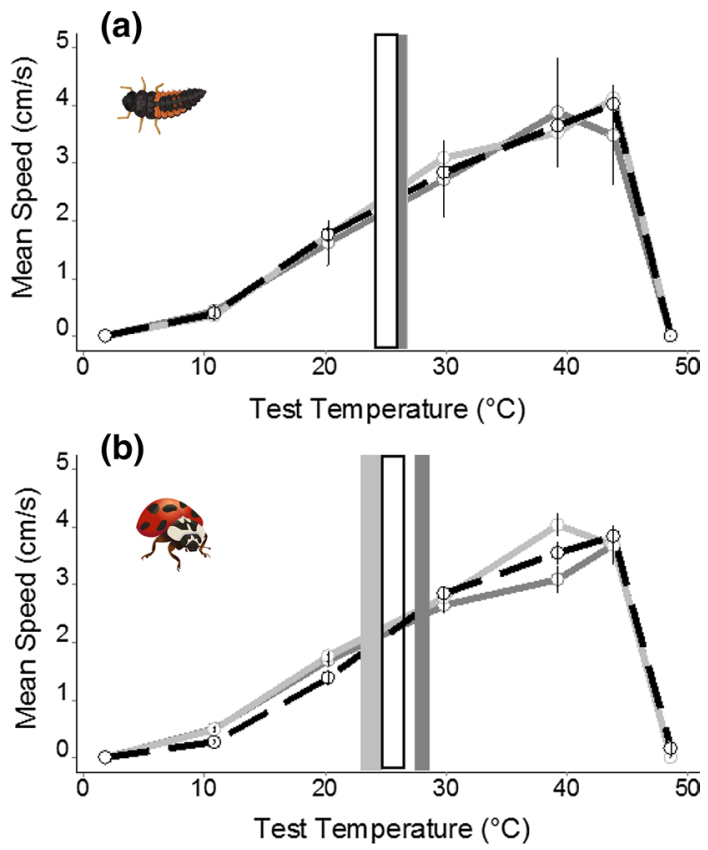

were exposed at the larval stage. Overall, larval temperature treatments resulted in higher adult $\mathrm{U}_{\max }$ than adult treatments $(t=3.01, p=0.003)$ (Fig. 1e).

Larval temperature treatments resulted in a lower adult $\mathrm{T}_{\mathrm{BR}}$ in the cold-acclimated group compared to the Medium or Warm groups $(t=2.31, p=0.03 ; t=2.38, p=0.02$ respectively), but Medium or Warm did not differ $(t=0.16, p=0.87)$ (Fig. 1f). There were no treatment effects on $\mathrm{T}_{\mathrm{BR}}$ in adults exposed at the adult stage (Fig. 1f). Males had higher $\mathrm{T}_{\mathrm{BR}}$ than females after exposure to treatments as larvae $(t=3.27, p<0.01)$, but had lower $\mathrm{T}_{\mathrm{BR}}$ than females after exposure as adults $(t=-2.48, p=0.02$; Fig. S6). Overall, there was no difference between the $\mathrm{T}_{\mathrm{BR}}$ of adults acclimated at the adult stage and larval stage $(t=-0.81, p=0.42)$.

\section{Fitness proxies}

The number of egg clutches did not vary among temperatures exposures within life stage groups and there were no significant differences between beetles exposed as adults and larvae overall $(\mathrm{z}=-1.4, \mathrm{p}=0.16)$ (Table $\mathrm{S} 1)$. However, the relationship between the total number of eggs (taken from the three first clutches) and body mass differed among acclimation regimes within life stage treatments: the total number of eggs from larvae treated at the Warm exposure was positively related to adult mass which differed from Cold and Medium exposures $(t=7.6, p<0.001 ; t=8.4, p<0.001$, respectively). By contrast, for beetles acclimated at the adult stage, the relationship between mass and number of eggs increased for Cold acclimation but decreased for the Medium acclimation $(t=-4.28$, $p<0.001)$. These interactions were highly significant when analysing data for both life stages together (Mass $\times$ Treatment $\times$ Life-stage interaction, $\mathrm{z}=5.52, p<0.0001$ ). After Warm acclimation of larvae, larger size beetles laid more eggs while this relationship was 
not maintained in the Cold treatment or in adult exposures (Cold and Warm). When beetles were treated at the larval stage, hatching success of their offspring increased from first to third clutch $(\mathrm{z}=3.86, p<0.001)$ and was higher for the Warm and Medium treatments than the Cold $(\mathrm{z}=2.28, p=0.02$ and $\mathrm{z}=4.2, p<0.001$, respectively), but these patterns were not found in adult-stage exposed beetles. Larval acclimations resulted in lower hatching success of eggs laid by treated females than adult acclimations overall $(\mathrm{z}=2.26, p=0.02)$. However, the composite index of fitness did not differ among acclimation regimes within larval or adult treatments $(t=1.3, p=0.20 ; t=-0.57, p=0.57$, respectively) nor the life stage of exposure $(\mathrm{t}=-0.27, p=0.8$; Fig. 3$)$.

\section{Discussion}

This study examined diverse, ecologically-relevant behavioural and physiological traits in a globally invasive beetle, Harmonia axyridis, to determine the relative importance of the timing of thermal acclimation. More specifically, we asked at what stage, larval or adult, does the thermal exposure manifests in the traits scored at adulthood. Within generations, predictable environments should favour developmental plasticity while varying and unpredictable environments should support reversible plasticity. We anticipated that the predictability of the thermal environment from larva to adulthood would be low given the higher mobility of adults compared to larvae, which could favour reversible adult plasticity. While the acclimation of larvae did not have lasting effects on the $\mathrm{CT}_{\max }, \mathrm{CT}_{\min }, \mathrm{T}_{\text {pref }}$, and walking $\mathrm{T}_{\mathrm{opt}}$ of adult beetles, larval exposures had an effect on $\mathrm{U}_{\max }$ and $\mathrm{T}_{\mathrm{BR}}$ of adult beetles. By contrast, we found adult plasticity of $\mathrm{CT}_{\max }$ and $\mathrm{T}_{\text {pref }}$ after specific treatments at adulthood. Therefore, this study demonstrates that $H$. axyridis exhibits distinct plastic responses for several performance traits following different life stage temperature treatments, but the patterns cannot be generalised across the two timings of exposure investigated here.

The finding that larval temperature exposure persists to affect adult performance in some traits but not others reinforces the need to assess acclimation effects for multiple traits and stages in insects with complex life cycles (Gray 2013; Mutamiswa et al. 2019). More importantly, these results suggest that the drivers (e.g. cues, environmental grain, opportunity costs, and associated risks) and perhaps mechanisms (e.g. biochemistry, genetics) of plasticity of temperature tolerance and selection are different to those shaping plasticity of walking speed. The relatively long lifespan of $H$. axyridis (estimated to be up to 150 days; Berkvens et al. 2008) means the environment can be highly variable within generations.

Fig. 3 Effects of larval and adult exposures to Cold, Medium and Warm temperature treatments (from left to right) on composite fitness of adults. Note: larval exposures to temperature treatments are shown on the left, adult exposures on the right; all individuals were females tested for fitness metrics during adulthood

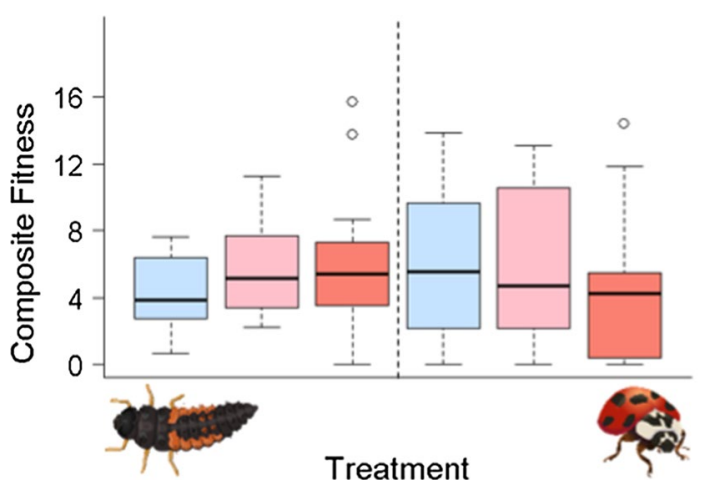

Treatment 
For example, larvae hatching in spring will experience much of their adulthood in warmer summer conditions. If the acclimation response to temperature during development was irreversible, and if there was a mismatch between the thermal environments during development and adulthood, or cues for predicting the future thermal environment were unreliable, an irreversible acclimation response would be a risky strategy (also see van Dyck et al. 2015). In these scenarios, reversible acclimation responses may be favoured (Gilchrist 1996; Ghalambor et al. 2007; Angilletta 2009). However, many components not measured here would be needed to generate robust predictions based on the degree of temperature predictability, including the assessment of microclimate and body temperature of larvae and adults in the field and relating these to detailed measurements of the time course of plastic responses in this species.

As opposed to shifts in maximal walking performance $\left(\mathrm{U}_{\max }\right)$, we found no plasticity in the $\mathrm{T}_{\text {opt }}$ for walking speed. Despite its potential fitness advantage, mechanisms underlying plasticity of $\mathrm{T}_{\mathrm{opt}}$ may be constrained, energetically expensive, or genes or cellular responses associated with plasticity may be linked to other genes conferring low fitness (see Angilletta 2009; Klepsatel et al. 2013; Gerken et al. 2016). No evidence of acclimation of running speed $\mathrm{T}_{\mathrm{opt}}$ (or jumping distance) was found in the cricket Acheta domesticus (Lachenicht et al. 2010), or in two terrestrial mite species, Halozetes fulvus and Podacarus auberti (Deere and Chown 2006), but the scarcity of examples limits the identification of any general evolutionary patterns or lack thereof.

The ability to alter $\mathrm{T}_{\text {pref }}$ through adult acclimation can have potential fitness benefits, enabling individuals to target temperatures that are most beneficial for a particular environment. Assuming $H$. axyridis selects temperatures that maximise fitness, and compensation for environmental change had occurred, Cold treatments should have led to a preference for cold temperatures and vice versa. Here, exposure to the Cold treatment in adulthood resulted in $H$. axyridis that selected warmer temperatures than after exposure to the Medium or Warm treatments. This could be an advantageous strategy in cooler seasons by enabling maximum utilisation of warm microsites during windows of opportunity and leading to faster metabolism or better performance in cold conditions (e.g. completion of development). It could also ensure optimal development of sperm and ova within sexually mature individuals. The reverse, where exposure to warm temperature cycles did not lead to an increase in $\mathrm{T}_{\text {pref }}$, may be protective because in warmer seasons there is a greater risk of over-heating, and operating at warm temperatures for longer periods carries rapidly increasing metabolic costs (and see discussions in Martin and Huey 2008; Basson and Clusella-Trullas 2015). Whether the potential fitness advantages of thermal acclimation of $\mathrm{T}_{\text {pref }}$ are realised depends largely on the stability, predictability and duration of the thermal environment experienced (Piersma and Drent 2003; Chown and Terblanche 2007; Angilletta 2009).

Several studies show that developmental effects can also be increasingly buffered with age (e.g. Gibert et al. 2001), meaning that any reversible physiological plasticity that may have occurred during exposure to temperature treatments as larvae can wear off and may have been undetectable when individuals were later tested as adults. However, the converse has been found too. Kellermann et al. (2017) showed that the effects of developmental temperatures on upper limits of Drosophila melanogaster were larger than adult acclimation effects, but the developmental acclimation treatments involved exposure to constant temperature regimes and lasted from egg to adulthood. This suggest that the intensity and duration of the acclimation regime influences the persistence of the response at the adult stage but also that the interpretations across species and studies are confounded by the thermal regimes used and how these relate to the 
life-history of the species investigated. Comparisons between different life stage exposures (significant for $\mathrm{U}_{\max }$ with larvae > adults) therefore need to be made with caution since acclimation regimes occurring at different life stages may not represent equivalent treatments, and therefore our main focus here is to highlight the distinct plastic responses resulting from different life stage exposures across a diverse suite of traits.

The mixed, trait-specific support for hypotheses about acclimation responses is not unique to this study and several examples exist showing similar complex findings (e.g. Kristensen et al. 2008; Clusella-Trullas et al. 2010). Despite a recent increase, the number of studies that examine thermal plasticity responses and long-lasting effects, both within and between generations, remains limited. In Plutella xylostella moths, extreme hot events that occurred closer to the adult stage had a more marked impact on reproductive output than those events occurring earlier in life, suggesting that the assessment of fitness costs depends on when the extreme thermal conditions occurred in its early life stages (Zhang et al. 2015). In our study, the significant effects of life stage exposure treatment on adult fitness also differed across fitness traits measured (e.g. number of clutches versus number of eggs). These effects balanced each other in a way that the composite fitness index which included all reproductive traits measured, did not differ between treatments. That is, larval and adult exposures resulted in a similar reproductive output in relative terms. However, this index did not incorporate all facets of an individual's lifetime fitness such as reproductive age-span, copulation and oviposition patterns, or fertility (Zhang et al. 2015; Porcelli et al. 2017). The traits measured in our study are likely to represent more immediate consequences of prior parental history than lifetime fecundity. Nonetheless, it would be suitable to consider additional fitness parameters to more comprehensively assess the potential costs of these distinct plastic responses.

This study has shown that acclimation at the adult stage resulted in flexible phenotypes of upper temperature tolerance and temperature preference but not of other traits, while exposures of earlier stages of development changed the height and breadth of the adult walking speed performance curve. These findings support some of the predictions relating to long term benefits of thermal acclimation in this species. Given that thermal acclimation can significantly alter the rates of genetic and phenotypic assimilation and hence, adaptive capacity (Sgrò et al. 2016), incorporating plastic responses resulting from these different life stage exposures to altered environments will be essential for predicting this species' distribution in future climate scenarios (Radchuk et al. 2013; Maino et al. 2016).

Acknowledgements We are grateful to E. Nortje, I.A. Minnaar and N. Mbongwa for laboratory assistance and the anonymous referees and Associate Editor for their helpful comments on an earlier version of the manuscript. The first author was supported by a Masters bursary from the DST - NRF Centre of Excellence for Invasion Biology, Stellenbosch University. Project funding was obtained from the DST - NRF Centre of Excellence for Invasion Biology.

Authors contribution SCT conceived the study and all authors refined experimental protocols; RS ran experiments and gathered data; RS and SCT performed analyses; all authors contributed to writing and gave final approval.

Availability of data and materials The datasets generated and analysed during the current study are available from the corresponding author on request.

\section{Compliance with ethical standards}

Conflict of interest The authors declare they have no conflict of interest. 
Ethical statement All applicable international, national, and/or institutional guidelines for the care and use of animals were followed.

\section{References}

Andersen JL, Manenti T, Sørensen JG, MacMillan HA, Loeschke V, Overgaard J (2015) How to assess Drosophila cold tolerance: chill coma temperature and lower lethal temperature are the best predictors of cold distribution limits. Funct Ecol 29:55-65

Angilletta MJ (2009) Thermal adaptation: a theoretical and empirical synthesis. Oxford University Press, Oxford

Bakken GS (1992) Measurement and application of operative and standard operative temperatures in ecology. Am Zool 32:194-216

Basson CH, Clusella-Trullas S (2015) The behavior-physiology nexus: behavioral and physiological compensation are relied on to different extents between seasons. Physiol Biochem Zool 88:384-394

Beaman JE, White CR, Seebacher F (2016) Evolution of plasticity: mechanistic link between development and reversible acclimation. Trends Ecol Evol 31:237-249

Berkvens N, Bonte J, Berkvens D, Deforce K, Tirry L, De Clercq P (2008) Pollen as an alternative food for Harmonia axyridis. Biocontrol 53:201-210

Bowler K (2005) Acclimation, heat shock and hardening. J Therm Biol 30:125-130

Bowler K, Terblanche JS (2008) Insect thermal tolerance: what is the role of ontogeny, ageing and senescence? Biol. Rev 83:339-355

Brown PMJ, Thomas CE, Lombaert E, Jeffries DL, Estoup A, Lawson Handley L-J (2011) The global spread of Harmonia axyridis (Coleoptera: Coccinellidae): distribution, dispersal and routes of invasion. Biocontrol 56:623-641

Cavieres G, Bogdanovich JM, Bozinovic F (2016) Ontogenetic thermal tolerance and performance of ectotherms at variable temperatures. J Evol Biol 29:1462-1468

Chevin L-M, Lande R (2015) Evolution of environmental cues for phenotypic plasticity. Evolution 69-10:2767-2775

Chown SL, Terblanche JS (2007) Physiological diversity in insects: ecological and evolutionary contexts. Adv Insect Physiol 33:50-152

Clusella-Trullas S, Chown SL (2014) Lizard thermal trait variation at multiple scales: a review. J Comput Physiol B 184:5-21

Clusella-Trullas S, Terblanche JS, Chown SL (2010) Phenotypic plasticity of locomotion performance in the seed harvester Messor capensis (Formicidae). Physiol Biochem Zool 83:519-530

Cotto O, Sandell L, Chevin L-M, Ronce O (2019) Maladaptive shifts in life history in a changing environment. Am Nat 194 (in press)

Crawley M (2007) The R Book. Wiley, Hoboken

Deere JA, Chown SL (2006) Testing the beneficial acclimation hypothesis and its alternatives for locomotor performance. Am Nat 168:630-644

Dillon ME, Liu R, Wang G, Huey RB (2012) Disentangling thermal preference and the thermal dependence of movement in ectotherms. J Therm Biol 37:631-639

Fischer K, Eenhoorn E, Bot ANM, Brakefield PM, Zwaan BJ (2003) Cooler butterflies lay larger eggs: developmental plasticity versus acclimation. Proc B R Soc 270:2051-2056

Fragata I, Lopes-Cunha M, Barbaro M, Kellen B, Lima M, Faria GS et al (2015) Keeping your options open: maintenance of thermal plasticity during adaptation to a stable environment. Evolution 70:195-206

Fusco G, Minelli A (2010) Phenotypic plasticity in development and evolution: facts and concepts. Philos Trans R Soc B 365:547-556

Gaston KJ, Chown SL (1999) Elevation and climatic tolerance: a test using dung beetles. Oikos 86:584-590

Gerken AR, Eller OC, Hahn DA, Morgan TJ (2015) Constraints, independence, and evolution of thermal plasticity: probing genetic architecture of long- and short-term thermal acclimation. Proc Natl Acad Sci USA 112:4399-4404

Ghalambor CK, McKay JK, Carroll SP, Reznick DN (2007) Adaptive versus non-adaptive phenotypic plasticity and the potential for contemporary adaptation in new environments. Funct Ecol 21:394-407

Gibert P, Huey RB, Gilchrist GW (2001) Locomotor performance of Drosophila melanogaster: interactions among developmental and adult temperatures, age and geography. Evolution 55:205-209

Gilchrist GW (1995) Specialists and generalists in changing environments. 1. Fitness landscapes of thermal sensitivity. Am Nat 146:252-270 
Gilchrist GW (1996) Quantitative genetic analysis of thermal sensitivity in the locomotor performance curve of Aphidius ervi. Evolution 50:1560-1572

Gray EM (2013) Thermal acclimation in a complex life cycle: The effects of larval and adult thermal conditions on metabolic rate and heat resistance in Culex pipiens (Diptera: Culicidae). J Insect Physiol 59:1001-1007

Grill CP, Moore AJ, Brodie ED III (1997) The genetics of phenotypic plasticity in a colonizing population of the ladybird beetle, Harmonia axyridis. Heredity 78:261-269

Hangartner S, Hoffmann A (2016) Evolutionary potential of multiple measures of upper thermal tolerance in Drosophila melanogaster. Funct Ecol 30:442-452

Kawai A (1976) Analysis of the aggregation behaviour in the larvae of Harmonia axyridis Pallas (Coleoptera: Coccinellidae) to prey colony. Res Populat Ecol 18:123-134

Kellermann V, van Heerwaarden B, Sgrò CM, Hoffmann AA (2009) Fundamental evolutionary limits in ecological traits drive Drosophila species distributions. Science 325:1244-1246

Kellermann V, Overgaard J, Loeschcke V, Kristensen TN, Hoffmann AA (2013) Trait associations across evolutionary time within a Drosophila phylogeny: correlated selection or genetic constraint? PLoS ONE 8:e72072

Kellermann V, van Heerwaarden B, Sgrò CM (2017) How important is thermal history? Evidence for lasting effects of developmental temperature on upper thermal limits in Drosophila melanogaster. Proc B R Soc 284:20170447

Kingsolver JG, Woods HA, Buckley LB, Potter KA, MacLean HJ, Higgins JK (2011) Complex life cycles and the responses of insects to climate change. Integr Comput Biol 51:719-732

Klepsatel P, Gáliková M, De Maio N, Huber CD, Schlötterer C, Flatt T (2013) Variation in thermal performance and reaction norms among populations of Drosophila melanogaster. Evolution 67:3573-3587

Knapp M (2014) Emergence of sexual size dimorphism and stage -specific effects of elevated temperature on growth date and development rate in Harmonia axyridis. Physiol Entomol. https://doi. org/10.1111/phen.12079

Knapp M, Nedvěd O (2013) Gender and timing during ontogeny matter: effects of a temporary high temperature on survival, body size and colouration in Harmonia axyridis. PLoS ONE 8:e74984

Kooi RE, Brakefield PM (1999) The critical period for wing pattern induction in the polyphonic tropical butterfly Bicyclus anynana (Satyrinae). J Insect Physiol 45:201-212

Kristensen TN, Hoffmann AA, Overgaard J, Sørensen JG, Hallas R, Loeschcke V (2008) Costs and benefits of cold acclimation in field-released Drosophila. Proc Natl Acad Sci USA 105:216-221

LaMana ML, Miller JC (1998) Temperature-dependent development in an Oregon population of Harmonia axyridis (Coleoptera: Coccinellidae). Environ Entomol 27:1001-1005

Lachenicht MW, Clusella-Trullas S, Boardman L, Le Roux C, Terblanche JS (2010) Effects of acclimation temperature on thermal tolerance, locomotion performance and respiratory metabolism in Acheta domesticus (Orthoptera: Gryllidae). J Insect Physiol 56:822-830

Lanzoni A, Accinelli G, Bazzocchi GG, Burgio G (2004) Biological traits and life table of the exotic Harmonia axyridis compared with Hippodamia variegata, and Adalia bipunctata (Col., Coccinellidae). J Appl Entomol 128:298-306

Levins R (1968) Evolution in changing environments: some theoretical expectations. Princeton University Press, Princeton

Liefting M, van Grunsven RHA, Morrisey MB, Timmermans MJTN, Ellers J (2015) Interplay of robustness and plasticity of life-history traits drives ecotypic differentiation in thermally distinct habitats. J Evol Biol 28:1057-1066

Lockwood BL, Gupta T, Scavotto R (2018) Disparate patterns of thermal adaptation between life stages in temperate vs. tropical Drosophila melanogaster. J Evol Biol 31:323-331

Lombaert E, Estoup A, Facon B, Joubard B, Gregoire J-C, Jannin A, Blin A, Guillemaud T (2014) Rapid increase in dispersal during range expansion in the invasive ladybird Harmonia axyridis. J Evol Biol 27:508-517

Martin TL, Huey RB (2008) Why "suboptimal” is optimal: Jensen's inequality and ectotherm thermal preferences. Am Nat 171:E102-E118

Maino JL, Kong JD, Hoffmann AA, Barton MG, Kearney MR (2016) Mechanistic models for predicting insect responses to climate change. Curr Opin Insect Sci 17:81-86

McCue MD, Boardman L, Clusella-Trullas S, Kleynhans E, Terblanche JS (2016) The speed and metabolic cost of digesting a blood meal depends on temperature in a major disease vector. J Exp Biol 219:1893-1902

Michie LJ, Mallard F, Majerus MEN, Jiggins FM (2010) Melanic through nature or nurture: genetic polymorphism and phenotypic plasticity in Harmonia axyridis. J Evol Biol 23:1699-1707 
Mutamiswa R, Machekano H, Chidawanyika F, Nyamukondiwa C (2019) Life-stage related responses to combined effects of acclimation temperature and humidity on the thermal tolerance of Chilo partellus (Swinhoe) (Lepidoptera: Crambidae). J Therm Biol 79:85-94

Nougué O, Svendsen N, Jabbour-Zahad R, Lenomand T, Chevin L-M (2016) The ontogeny of tolerance curves: habitat quality vs. acclimation in a stressful environment. J Anim Ecol 85:1625-1635

Nyamukondiwa C, Terblanche JS (2010) Within-generation variation of critical thermal limits in adult Mediterranean and Natal fruit flies Ceratitis capitata and Ceratitis rosa: thermal history affects short-term responses to temperature. Physiol Entomol 35:255-264

Overgaard J, Kearney M, Hoffmann AA (2014) Sensitivity to thermal extremes in Australian Drosophila implies similar impacts of climate change on the distribution of widespread and tropical species. Glob Chang Biol 20:1738-1750

Osawa N (2000) Population field studies on the aphidophagous ladybird beetle Harmonia axyridis (Coleoptera: coccinnellidae): resource tracking and population characteristics. Popul Ecol 42:115-127

Piersma T, Drent J (2003) Phenotypic flexibility and the evolution of organismal design. Trends Ecol Evol 18:228-233

Pigliucci M, Murren CJ, Schlichting CD (2006) Phenotypic plasticity and evolution by genetic assimilation. J Exp Biol 209:2362-2367

Porcelli D, Gaston KJ, Butlin RK, Snook RR (2017) Local adaptation of reproductive performance during thermal stress. J Evol Biol 30:422-429

Potter KA, Davidowitz G, Woods HA (2011) Cross-stage consequences of egg temperature in the insect Manduca sexta. Funct Ecol 25:548-556

Purse BV, Comont R, Butler A, Brown PMJ, Kessel C, Roy HE (2015) Landscape and climate determine patterns of spread for all colour morphs of the alien ladybird Harmonia axyridis. J Biogeogr 42:575-588

Raak-van den Berg CL, Hemerik L, van der Werf W, de Jong PW, van Lenteren JC (2017) Life history of the harlequin ladybird, Harmonia axyridis: a global meta-analysis. Biocontrol 62:283-296

Radchuk V, Turlure C, Schtickzelle N (2013) Each life stage matters: the importance of assessing the response to climate change over the complete life cycle in butterflies. J Anim Ecol 82:275-285

Rako L, Hoffmann AA (2006) Complexity of the cold acclimation response in Drosophila melanogaster. J Insect Physiol 52:94-104

Roy HE, Brown PMJ, Adriaens T, Berkvens N, Borges I, Clusella-Trullas S et al (2016) The harlequin ladybird, Harmonia axyridis: global perspectives on invasion history and ecology. Biol Invasions 18:997-1044

Seebacher S (2005) A review of thermoregulation and physiological performance in reptiles: what is the role of phenotypic flexibility? J Comput Physiol B 175:453-461

Schulte PM, Healy TM, Fangue NA (2011) Thermal performance curves, phenotypic plasticity, and the time scales of temperature exposure. Integr Comput Biol 51:691-702

Sgrò CM, Terblanche JS, Hoffmann AA (2016) What can plasticity contribute to insect responses to climate change? Annu Rev Entomol 61:433-451

Sinclair BJ, Williams CW, Terblanche JS (2012) Variation in thermal performance among insect populations. Physiol Biochem Zool 85:594-606

Slotsbo S, Schou MF, Kristensen TN, Loeschcke V, Sørensen JG (2016) Reversibility of developmental heat and cold plasticity is asymmetric and has long-lasting consequences for adult thermal tolerance. $\mathrm{J}$ Exp Biol 219:2726-2732

Sørensen JG, Kristensen TN, Overgaard J (2016) Evolutionary and ecological patterns of thermal acclimation capacity in Drosophila: is it important for keeping up with climate change? Curr Opin Insect Sci 17:98-104

Terblanche JS, Chown SL (2006) The relative contributions of developmental plasticity and adult acclimation to physiological variation in the tsetse fly, Glossina pallidipes (Diptera, Glossinidae). J Exp Biol 209:1064-1073

Uno H, Stillman JH (2020) Lifetime eurythermy by seasonally matched thermal performance of developmental stages in an annual aquatic insect. Oecologia. https://doi.org/10.1007/s00442-020-04605-z

Van Dyck H, Bonte D, Puls R, Gotthard K, Maes D (2015) The lost generation hypothesis: could climate change drive ectotherms into a developmental trap? Oikos 124:54-61

Via S, Lande R (1985) Genotype-environment interaction and the evolution of phenotypic plasticity. Evolution 39:505-522

Weinig C, Delph L (2001) Phenotypic plasticity early in life constrains developmental responses later. Evolution 55:930-936

Weldon CW, Terblanche JS, Chown SL (2011) Time-course for attainment and reversal of acclimation to constant temperature in two Ceratitis species. J Therm Biol 36:479-485 
West-Eberhard MJ (2003) Developmental plasticity and evolution. Oxford University Press, New York Westneat DF, Potts LJ, Sasser KL, Shaffer JD (2019) Causes and consequences of phenotypic plasticity in complex environments. Trends Ecol Evol 34:555-568

Wilson RS, Franklin CE (2002) Testing the beneficial acclimation hypothesis. Trends Ecol Evol 17:66-70

Zhang S, Cao Z, Wang Q, Zhang F, Liu T-X (2014) Exposing eggs to high temperatures affects the development, survival and reproduction of Harmonia axyridis. J Therm Biol 39:40-44

Zhang W, Rudolf VHW, Ma C-S (2015) Stage-specific heat effects: timing and duration of heat waves alter demographic rates of a global insect pest. Oecologia 179:947-957

Zeilstra I, Fischer K (2005) Cold tolerance in relation to developmental and adult temperature in a butterfly. Physiol Entomol 30:92-95

Publisher's Note Springer Nature remains neutral with regard to jurisdictional claims in published maps and institutional affiliations. 